\title{
Some more exact results concerning multifield moduli of two-phase composites
}

\author{
Mordehai Milgrom \\ Department of Condensed-Matter Physics, Weizmann Institute of Science 76100 Rehovot, Israel
}

\begin{abstract}
Chen (1996) has recently shown how the response matrix of a two-phase composite can be written as certain linear combinations of products of the component matrices. We elaborate on Chen's expansions by deriving them in a different way, which a. shows them in a different light, and b. permits us to generalize them further. As an application of our results we find exact microstructure-independent relations between the moduli of the two components and those of any composite. The body of these relations is equivalent to the compatibility relations of Milgrom and Shtrikman (1989a), but they are cast in a rather different form, which has certain advantages. As an example, we show how any modulus of an arbitrary two-phase composite can be written in closed form as a linear combination of any other $n$ of its moduli, with coefficients that depend only on the component moduli, but not on the volume fractions, or the microstructure.
\end{abstract}

\section{Introduction}

Our discussion concerns the response of a medium to many coupled fields, such as in the thermoelectric phenomenon, in the magneto-electric effect, or in coupled, multispecies diffusion. We concentrate of the response properties of a composite-whose multifield linear-response matrix is $L^{*}$-made of two components with response matrices $L_{a}, L_{b}$. Chen (1996), using a matrix-decomposition scheme, has recently shown that one can write e.g.

$$
L^{*}=a_{0} L_{b}+a_{1} L_{a}+a_{2} L_{a} L_{b}^{-1} L_{a}+\ldots+a_{n-1} L_{a} L_{b}^{-1} L_{a} \ldots L_{b}^{-1} L_{a}
$$

with $L_{a}$ appearing $n-1$ times in the last term, were $n$ is the number of coupled fields involved. Chen derives several other similar expansions, and there are similar expansions for $\left(L^{*}\right)^{-1}$. The expansion coefficients are functions of the detailed makings of the composite-viz. the volume fractions, and the microstructure.

We rederive and generalize Chen's expansions in $\S 2$, and determine their coefficients in $\S 3$. In $\S 4$ we present a new, determinant form for the compatibility relations of Milgrom and Shtrikman (1989a). 


\section{Rederivation and generalization of Chen's expansions}

Following Milgrom and Shtrikman (1989a) we take $W$ to be a (non-singular) matrix that diagonalize $L_{a}$ and $L_{b}$ simultaniously by a congruence transformation, such that

$$
W L_{a} \tilde{W}=\lambda, \quad W L_{b} \tilde{W}=I
$$

where $\lambda=\operatorname{diag}\left(\lambda_{1}, \ldots, \lambda_{n}\right)$ is diagonal, and $I$ is the unit matrix. Such a matrix always exists as $L_{a}, L_{b}$ are symmetric and positive definite. As explained in Milgrom and Shtrikman (1989a), the physics of the problem dictates that the response matrix, $L^{*}$, of any composite material of the two components is also digonalized by $W$ :

$$
W L^{*} \tilde{W}=\lambda^{*}=\operatorname{diag}\left(\lambda_{1}^{*}, \ldots, \lambda_{n}^{*}\right) .
$$

Consider now the series of $n \times n$ matrices $P^{(k)}$, for $k$ an arbitrary integer, such that $P^{(k)}$ is transformed by $W$ into $\lambda^{k}$-the $k$ th power of $\lambda$ :

$$
P^{(k)} \equiv W^{-1} \lambda^{k} \tilde{W}^{-1} .
$$

It is easy to see from eq.(2) that $P^{(0)}=L_{b}, P^{(1)}=L_{a}$, and, in general,

$$
P^{(k)}=L_{b}\left(L_{b}^{-1} L_{a}\right)^{k}
$$

Thus, for positive $k P^{(k)}=L_{a} L_{b}^{-1} L_{a} \ldots L_{b}^{-1} L_{a}$, and for negative $k=-|k| P^{(k)}=L_{b} L_{a}^{-1} L_{b} \ldots L_{a}^{-1} L_{b}$, with $L_{a}$, or $L_{a}^{-1}$ appearing $|k|$ times. If one interchanges the role of $L_{a}$ and $L_{b}$ (W is than changed): $P^{(k)}\left(L_{a}, L_{b}\right)=P^{(-k+1)}\left(L_{b}, L_{a}\right)$.

Let $N$ be the number of different $\lambda_{i}$ s, than it is clear that:

a. Any set of more than $N$ integer powers of the matrix $\lambda$ are linearly dependent (because each can be viewed as a vector of length $N$ ).

b. Any set of $N$ different (integer) powers, $\lambda^{k_{1}}, \ldots, \lambda^{k_{N}}$ are linearly independent. This is because the determinant of the matrix

$$
M(\vec{\lambda}, \vec{k}) \equiv\left(\begin{array}{cccc}
\lambda_{1}^{k_{1}} & \cdot & \cdot & \lambda_{1}^{k_{N}} \\
\cdot & \cdot & \cdot \\
\cdot \dot{k_{1}} & & \cdot & \cdot \\
\lambda_{N}^{k_{N}} & \cdot & \cdot & \lambda_{N}^{k_{N}}
\end{array}\right)
$$

does not vanish unless at least two of the $\lambda_{i}$ are equal (for the case of consecutive powers this is just proportional to the Vandermonde determinant-see below)(see e.g. Aitken 1949]).

c. Any $n \times n$ diagonal matrix, with the same equalities among its element as in $\lambda$, can be written as a linear combination of any set of $N$ different powers of $\lambda$. Property c. is satisfied, in particular, by $\lambda^{*}$ for any composite, by its inverse, or, for that matter, by any integer power of it. 
For the sake of clarity we assume from now on that $\lambda$ is non-degenerate i.e. $N=n$; all we say below goes though straightforwardly for $N<n$.

Properties a.-c. are carried through by transforming back with $W$, whereby $\lambda^{k}$ goes back to $P^{(k)}, f(\lambda)$ is carried back to $f\left(L_{a} L_{b}^{-1}\right) L_{b}=L_{b} f\left(L_{b}^{-1} L_{a}\right), \lambda^{*}$ goes to $L^{*},\left(\lambda^{*}\right)^{-1}$ goes to $L_{b}\left(L^{*}\right)^{-1} L_{b}$, $\left(\lambda^{*}\right)^{m}$ ( $m$ positive) goes to $L^{*} L_{b}^{-1} L^{*} \ldots L_{b}^{-1} L^{*}$ ( $L^{*}$ appearing $m$ times), etc. We thus have

a'. Any set of more than $n$ of the $P^{(k)}$ matrices are linearly dependent.

b'. Any set of $n$ different $P^{(k)}$ s are linearly independent.

c'. Any of the matrices, such as $L^{*}, L_{b}\left(L^{*}\right)^{-1} L_{b}$, etc. can be written as a linear combination of any $n$ of the $P^{(k)} \mathrm{s}$ (any $N$ when $N<n$ ).

So, for example, for every choice of different powers there are coefficients $a_{i}$ such that

$$
L^{*}=\sum_{i=1}^{n} a_{i} P^{\left(k_{i}\right)} .
$$

Chen's expansions are special cases of eq.(17) [and an analogous one for $L_{b}\left(L^{*}\right)^{-1} L_{b}$ ] with consecutive values of $k_{i}: k=k_{0}, k_{0}+1, \ldots, k_{0}+n-1$, and special values of $k_{0}$. For example, eq.(11) is obtained when expanding $L^{*}$ with $k_{0}=0$. Expanding $L_{b}\left(L^{*}\right)^{-1} L_{b}$ using $k_{0}=-n+1$ gives

$$
\left(L^{*}\right)^{-1}=b_{0} L_{b}^{-1}+b_{1} L_{a}^{-1}+b_{2} L_{a}^{-1} L_{b} L_{a}^{-1}+\ldots+b_{n-1} L_{a}^{-1} L_{b} \ldots L_{b} L_{a}^{-1},
$$

another of Chen's expansions.

Obviously, instead of using the $P^{(k)}$ s as expansion basis, we could use the response matrices of different composites of the same two composites, or different space-space moduli matrices of anisotropic composites of the same components. This will give relations between different composites [for more details see the discussion in Milgrom and Shtrikman (1989a)].

\section{The expansion coefficients}

The expansion coefficients $a_{i}$ depend on the exact nature of the composite. It is sometimes useful to express these coefficients in terms of $\lambda_{i}$ and $\lambda_{i}^{*}$, which Chen (1996) does using a matrix decomposition theorem. In the present formalism the $a_{i}$ are just the coefficients of the linear expansion of the $n$-vector $\vec{\lambda}^{*}$, made of the diagonal of $\lambda^{*}$, in the $n n$-vectors made of the diagonals of $\lambda^{k_{i}}$. The vector $\vec{a}=\left(a_{1}, \ldots, a_{n}\right)$ is thus the solution of the linear equations

$$
M(\vec{\lambda}, \vec{k}) \vec{a}=\vec{\lambda}^{*}
$$

where $M$ is the matrix defined in eq.(6). [Their ilk are treated under 'alternant matrices' in Aitken 1949) (p. 111)] 
The case of consecutive $k \mathrm{~s}$ lands itself to further simplification: When $\vec{k}=\left(k_{0}, k_{0}+1, \ldots, k_{0}+n-1\right)$ eq.(9) can be written as

$$
V(\vec{\lambda}) \vec{a}^{\left(k_{0}\right)}=\vec{\delta}^{*}
$$

where $V$ is the Vandermonde matrix:

$$
V(\vec{\lambda}) \equiv\left(\begin{array}{cccccc}
1 & \lambda_{1} & \lambda_{1}^{2} & \cdot & \cdot & \lambda_{1}^{n-1} \\
1 & \lambda_{2} & \lambda_{2}^{2} & \cdot & \cdot & \lambda_{2}^{n-1} \\
\cdot & \cdot & \cdot & \cdot & & \cdot \\
\cdot & \cdot & \cdot & & \cdot & \cdot \\
1 & \lambda_{n} & \lambda_{n}^{2} & . & . & \lambda_{n}^{n-1}
\end{array}\right),
$$

and $\delta_{i}^{*} \equiv \lambda_{i}^{*} / \lambda_{i}^{k_{0}}$. Thus

$$
a_{i}^{\left(k_{0}\right)}=\sum_{j}\left(V^{-1}\right)_{i j} \lambda_{j}^{*} /\left(\lambda_{j}\right)^{k_{0}} .
$$

The elements of $V^{-1}$ can be written in closed form:

$$
\left(V^{-1}\right)_{i j}=(-1)^{i+j}\left|V_{n}\right|^{-1}\left|V_{n-1}^{j}\right| S_{n-i}\left(\lambda_{1}, \ldots, \lambda_{j-1}, \lambda_{j+1}, \ldots, \lambda_{n}\right) .
$$

Here, $\left|V_{n}\right|$ is the Vandermonde determinant for the $n \lambda_{i},\left|V_{n-1}^{j}\right|$ is the same for the $n-1 \lambda_{\mathrm{s}}$ excluding $\lambda_{j}$, and $S_{n-i}$ is the elementary symmetric polynomial of degree $n-i$ in its variables (here $\lambda_{1}, \ldots, \lambda_{j-1}, \lambda_{j+1}, \ldots, \lambda_{n}$ ); it is simply the sum of all possible products of $n-i$ subsets of different variables. So, for example, $S_{0}=1, S_{1}=\sum_{k \neq j} \lambda_{k}$, and $S_{n-1}=\lambda_{1} \lambda_{2} \ldots \lambda_{j-1} \lambda_{j+1} \ldots \lambda_{n}$. All this gives the expressions Chen (1996) obtains for the special values of $k_{0}$ corresponding to his expansions.

\section{A new form of the compatibility relations}

The fact underlying many an exact relation for two-phase composites is that only $n$ numbers are needed to specify all the the $n(n+1) / 2$ moduli of a response matrix. Thus, the off-diagonal elements of eq.(3) can be viewed as $n(n-1) / 2$ linear relations in the elements of $L^{*}$ with coefficients involving only the component moduli (through $W$ ). A given set of the compatibility relations of Milgrom and Shtrikman (1989a) is another, and more useful, set of $n(n-1) / 2$ relations between the moduli. In Chen's expansions and all their generalization discussed above [eq.(77)] the $n$ structure-dependent parameters are the expansion coefficients. While all these sets of constraints are equivalent, each has its advantages. We now proceed to derive yet other manifestations of the same constraints, which have their own advantages.

Let $A^{1}, \ldots, A^{n+1}$ be any $n+1$ matrices that become diagonal when transformed with $W$. They

can be any of the $P^{(k)}$ s, the response matrices of any isotropic composite of the two components, 
the space-space response matrix of an anisotropic composite, or appropriate products of such matrices of the form $M_{1} M_{2}^{-1} M_{3} \ldots M_{m-1}^{-1} M_{m}$, where the $M$ s are diagonalized by $W$. The arguments of $\S 1$ show that these $n+1$ matrices are linearly dependent. Thus, if we choose any set of $n+1$ matrix slots: $r_{1} s_{1}, \ldots, r_{n+1} s_{n+1}$, the $n+1$ vectors $\vec{a}_{i}=\left[A_{r_{1} s_{1}}^{i}, \ldots, A_{r_{n+1} s_{n+1}}^{i}\right], \quad(1 \leq i \leq n+1)$, are linearly dependent:

$$
\left|\begin{array}{ccc}
A_{r_{1} s_{1}}^{1} & \cdot & A_{r_{n+1} s_{n+1}}^{1} \\
\cdot & \cdot & \cdot \\
A_{r_{1} s_{1}}^{n+1} & \cdot & A_{r_{n+1} s_{n+1}}^{n+1}
\end{array}\right|=0 .
$$

Take, for example, for the above $n+1$ matrices any $n$ different $P^{(k)} \mathrm{s}$ with $k=k_{1}, \ldots, k_{n}$, and the response matrix, $L^{*}$, of some isotropic composite. Then eq.(14) tells us that

$$
\left|\begin{array}{ccccc}
L_{r_{1} s_{1}}^{*} & L_{r_{2} s_{2}}^{*} & \cdot & \cdot & L_{r_{n+1} s_{n+1}}^{*} \\
P_{r_{1} s_{1}}^{\left(k_{1}\right)} & P_{r_{2} s_{2}}^{\left(k_{1}\right)} & . & . & P_{r_{n+1} s_{n+1}}^{\left(k_{1}\right)} \\
\cdot & \cdot & \cdot & & \cdot \\
\dot{\left.k_{n}\right)} & \dot{\left(k_{n}\right)} & & \cdot & \\
P_{r_{1} s_{1}}^{\left(k_{n}\right.} & P_{r_{2} s_{2}}^{\left(k_{n}\right)} & \cdot & . & P_{r_{n+1} s_{n+1}}^{(}
\end{array}\right|=0,
$$

for any choice of different matrix slots $r_{i} s_{i}$.

Equation (15) constitute a linear relation, with structure-independent coefficients, between any chosen set of $n+1$ composite moduli. Any set of independent $n(n-1) / 2$ such determinant relations is equivalent to one matrix compatibility relation of Milgrom and Shtrikman (1989a) of the form

$$
L^{*} L_{a}^{-1} L_{b}=L_{b} L_{a}^{-1} L^{*}
$$

which embodies the same number of independent relations between the elements of $L_{a}, L_{b}$, and $L^{*}$. The advantage of the latter is that it involves only elements of the products $L_{a}^{-1} L_{b}$, and $L_{b} L_{a}^{-1}$, while the former involves higher-order products $P^{(k)}$. The advantage of the former is that each of its relations involve only $n+1$ of the elements of $L^{*}$, and they can be chosen at will, while in the latter each relation involves generically $2 n-1$ elements of $L^{*}$, which cannot be chosen at will. The new relations eq.(15) thus permit us to write any modulus of $L^{*}$ as a microstructure-independent linear combination of any other $n$ of its moduli. (Clearly, if we replace $L^{*}$ in eq. (15) by another of the $P^{(k)}$ s we get an identity, as the derivation of such a relation involves no physical arguments.)

One interesting example is the choice with $n$ of the slots being the diagonal ones, and the $(n+1)$ th some off-diagonal element $r s$. This gives a determinant relation of the form

$$
\left|\begin{array}{ccccc}
L_{r s}^{*} & l_{1}^{*} & \cdot & \cdot & l_{n}^{*} \\
P_{r s}^{\left(k_{1}\right)} & p_{1}^{\left(k_{1}\right)} & \cdot & \cdot & p_{n}^{\left(k_{1}\right)} \\
\cdot & \cdot & \cdot & & \cdot \\
P_{r s}^{\left(k_{n}\right)} & p_{1}^{\left(k_{n}\right)} & \cdot & \cdot & \cdot \\
\left(\dot{k}_{n}\right)
\end{array}\right|=0
$$


where $l_{i}^{*}$ are the diagonal coefficients of $L^{*}$, and $p_{j}^{\left(k_{i}\right)}$ is the $j$ th diagonal element of $P^{\left(k_{i}\right)}$. This form permits us to express all the off-diagonal moduli of any composite in terms of the diagonal moduli (and those of the components):

$$
L_{r s}^{*}=\sum_{i} \alpha_{i}^{r s} l_{i}^{*}, \text { where } \alpha_{i}^{r s} \equiv \frac{U_{i}^{(r s)}}{|P|} .
$$

Here, $|P|$ is the determinant of the diagonal elements $p_{j}^{\left(k_{i}\right)}$, and $U_{i}^{(r s)}$ are the appropriate cofactors, and all are calculated from the component moduli. An off-diagonal modulus of an arbitrary composite is then given by a universal linear function of the diagonal moduli of this composite.

For the special case when only two coupled fields are involved Milgrom and Shtrikman (1989a) were, in fact, able to write the single compatibility relation in determinant form (in this case $2 n-1=n+1)$. Our present result is a generalization to the $n>2$ case.

Equation(18) can be useful, for instance, in the following way: If all cross moduli are small and can be neglected to lowest order, one can find simultaneous bounds on the diagonal elements $l_{i}^{*} \approx \lambda_{i}^{*}$ : A region $B$ in the space of $\left(l_{1}^{*}, \ldots, l_{n}^{*}\right)$. Equation(18), with the left-hand side a parameter, defines a family of hyperplanes in this space. Bounds on individual $L_{r s}^{*}$ can then be found by identifying the two extreme hyperplanes of the family still touching the region $B$. Note that these would constitute (approximate) bounds on individual cross moduli. In contrast, the bounds discussed by Milgrom and Shtrikman (1989c), and by Chen (1996), are exact but involve combinations of many moduli.

All our results are applicable also to polycrystals made of a uniaxial single crystal as is evident from the discussion of Milgrom and Shtrikman (1989b).

I thank Tungyang Chen for permission to use and quote his results before publication.

\section{REFERENCES}

Aitken, A.C. 1949, Determinants and Matrices, Oliver and Boyd, Edinburgh.

Chen, T. 1996, Submitted to J. Mech. Phys. Solids

Milgrom, M. and Shtrikman, S. 1989a, Phys. Rev. A 40, 1568.

Milgrom, M. and Shtrikman, S. 1989b, Phys. Rev. B 40, 5991.

Milgrom, M. and Shtrikman, S. 1989c, J. App. Phys. 66, 3429.

This preprint was prepared with the AAS $\mathrm{LAT}_{\mathrm{E}} \mathrm{X}$ macros v3.0. 